\title{
Yeast systems biology in understanding principles of physiology underlying complex human diseases
}

\author{
Yu, Rosemary; Nielsen, Jens
}

Published in:

Current Opinion in Biotechnology

Link to article, DOI:

10.1016/j.copbio.2019.11.021

Publication date:

2020

Document Version

Peer reviewed version

Link back to DTU Orbit

Citation (APA):

Yu, R., \& Nielsen, J. (2020). Yeast systems biology in understanding principles of physiology underlying complex human diseases. Current Opinion in Biotechnology, 63, 63-69. https://doi.org/10.1016/j.copbio.2019.11.021

\section{General rights}

Copyright and moral rights for the publications made accessible in the public portal are retained by the authors and/or other copyright owners and it is a condition of accessing publications that users recognise and abide by the legal requirements associated with these rights.

- Users may download and print one copy of any publication from the public portal for the purpose of private study or research.

- You may not further distribute the material or use it for any profit-making activity or commercial gain

- You may freely distribute the URL identifying the publication in the public portal 


\section{Yeast systems biology in understanding 2 principles of physiology underlying 3 complex human diseases}

4

5

6

7

8

9

10

11

Rosemary $\mathrm{Yu}^{1,2}$ and Jens Nielsen ${ }^{1,2,3,4, *}$

${ }^{1}$ Department of Biology and Biological Engineering, Chalmers University of Technology, SE-412 96 Gothenburg, Sweden.

${ }^{2}$ Novo Nordisk Foundation Center for Biosustainability, Chalmers University of Technology, SE-412 96 Gothenburg, Sweden.

${ }^{3}$ Novo Nordisk Foundation Center for Biosustainability, Technical University of Denmark, DK-2800 Kgs. Lyngby, Denmark.

${ }^{4}$ Biolnnovation Institute, Ole Måløes Vej 3, DK-2200 Copenhagen N, Denmark.

*Corresponding author: Nielsen, Jens (nielsenj@chalmers.se )

\section{Highlights}

- Yeast is widely used to study cellular processes underlying complex human diseases

- Yeast systems biology provides holistic insights into disease-associated processes

- Findings in yeast systems biology are readily transferrable to human cell models

- Omics data integration into GEMs can accelerate advances in personalized medicine

\section{Abstract}

Complex human diseases commonly arise from deregulation of cell growth, metabolism, and/or gene expression. Yeast is a eukaryal model organism that is widely used to study these processes. Yeast systems biology benefits from the ability to exert fine experimental control over the cell growth rate and nutrient composition, which allows orthogonal experimental design and generation of multi-omics data at high resolution. This has led to several insights on the principles of cellular physiology, including many cellular processes associated with complex human diseases. Here we review these biological insights together with experimental and modeling approaches developed in yeast to study systems biology. The role of yeast systems biology to further advance systems and personalized therapies for complex diseases are discussed. 
Introduction

Saccharomyces cerevisiae is a widely used eukaryal model organism that has been instrumental for studying many pathways that are associated with human disease development. For many years yeast has been the go-to organism in functional genomics studies due to the ease by which the yeast genome could be manipulated, and the high degree of conservation in both protein function and regulatory circuitry between yeast and human cells [1]. Knowledge gained by studying yeast in an unbiased approach, eg through a genome-wide screen, can quickly be transferred to humans by confirming the finding(s) via a targeted approach in a human cell model, or by consistent observations in disease symptoms or clinical trials [1]. As such, yeast has been used to model many conditions/processes leading to complex diseases, most notably for several hallmarks of cancer (eg cell cycle control [2•], programmed cell death [3], genome instability [4]); different types of metabolic disorders (eg cholesterol metabolism [5], diabetes [6], mitochondrial dysfunction [7]); factors influencing aging (eg dietary restriction [8]); and etiology of neurodegenerative diseases (amyloid pathologies [9] and lysosomal storage disorders [10]).

At the systems level, many of these multi-factorial diseases arise from a deregulation of one or a combination of three key aspects of cell biology: cell growth, metabolism, and gene expression. While most of the individual components for each of these processes have been identified, currently we lack an understanding of how physiology is shaped by them in a holistic way. To this end, yeast has been used to pioneer the field of systems biology, in the same way that yeast molecular biology paved way for similar studies in human cells $[1,11]$. Using yeast as a model organism, new concepts and tools have been developed for top-down integration of multi-omics data into mathematical models, which allows cellular functions to be simulated in silico. Here, we review the principles of physiology that were discovered using yeast systems biology, which has given insight into the mechanisms of complex diseases in human cells. We also discuss new modeling approaches developed in yeast that will accelerate both human systems biology research and the development of systems and personalized therapeutic interventions.

\section{Cell growth}

In a basic model of cell growth, duplication of a cell entails duplication of each of its components [12]. The single largest macromolecular component of the cell biomass is protein, which in yeast represents $40-60 \%$ of dry mass depending on the growth condition; while RNA and DNA represent 8$10 \%$ and $0.1 \%$ of the yeast dry mass, respectively (Fig $1 \mathrm{~A}$ ) [13]. Moreover, protein is the only component that is self-replicating via ribosomes, which places a natural limitation on the cell growth rate. Thus it has long been proposed that cell growth is limited by protein synthesis, and many 
"classic" models of cell growth were formulated based on the self-replicating nature of ribosomes [14-16]. Indeed, in bacteria it has been shown that cells finely tune their ribosomal protein content to precisely match the protein synthesis demand at any given growth rate [17]. However, to directly test this hypothesis in fast-growing eukaryal cells such as yeast or human cancer cells, it is necessary to globally profile the expression dynamics of proteins under highly controlled experimental conditions, which has only recently been accomplished in yeast. Yeast is particularly suitable for these types of experiments, because the growth of yeast cells can be controlled very precisely and maintained at steady-state, which is often an important assumption in these models [1]. By growing

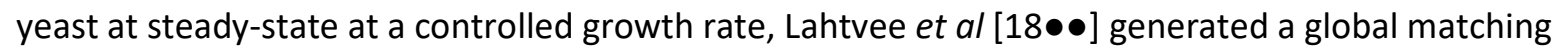
dataset that includes transcript abundance, protein abundance, protein degradation rates, and calculated protein synthesis rates. Integrative analysis of this data shows that protein synthesis rate matches closely with the rate of protein dilution from cell division, and degradation rate contributes

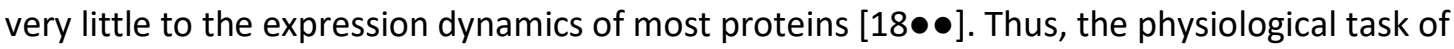
replicating the proteome places a global constraint on how fast cell replication can occur as a whole. In contrast, multiple transcript expression dynamics datasets $[19,20]$ have shown that globally the mRNA synthesis rate is much faster than the dilution rate, and most mRNAs are synthesized and degraded several times in a cell cycle. However, when growth conditions are experimentally modified to restrict phosphate availability (a critical component of nucleotides), the control of

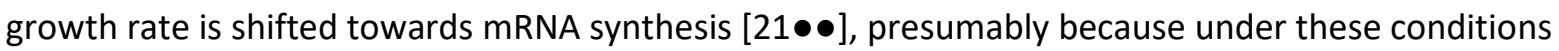
it becomes slower to accumulate mRNA to the point of duplication (Fig 1B). From an evolutionary perspective, it has been shown that the abundance of orthologous proteins is highly conserved between S. cerevisiae and S. pombe (across 400 million years of evolution), while protein degradation rates have significantly diverged [22], indicating that protein abundance is constrained by a strong evolutionary pressure which likely arose from the pressure to maintain a fast growth rate. In contrast, most of the orthologous genes between S. cerevisiae and S. paradoxus (across 5-10 million years of evolution) have diverged in mRNA abundance [23] as well as mRNA synthesis and degradation rates [24], in line with the idea that growth rate is not limited by mRNA abundance and thus there is less selection pressure to conserve transcript expression in evolution.

As yeast systems biology affirms that growth rate is limited by protein synthesis, intuitively growth rate would be maximized when cells only express the minimum amount of proteins necessary to carry out all cell functions. However, in most instances cells employ a trade-off and maintain excess proteins as reserves, while growing at a rate that is lower than the theoretical maximum $[25 \bullet \bullet, 26 \bullet]$. The size of the protein reserves underlies how quickly cells can respond to changing environments [26•]. For pharmaceuticals that target endogenous enzymes or processes, the sizes of 
specific protein reserves may also define the therapeutic window of the drug, whereby the same amount of a drug (eg an enzyme inhibitor) affects the growth of disease cells, but healthy cells remain robust due to the presence of excess enzyme capacity [27]. Thus, quantifying the amount of protein reserves in normal and diseased cells will have significant impact on the design and testing of pharmaceuticals. Recently several approaches have been taken to estimate the protein reserves of various processes in yeast. By generating high-resolution proteomic datasets of yeast growing at

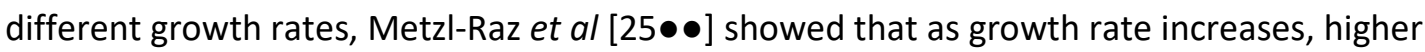
proportions of the proteome are allocated to ribosomal proteins and enzymes in amino acid biosynthesis, while lower proportions are allocated to enzymes in central carbon metabolism (CCM). This indicates that at lower growth rates, cells maintain high amounts of CCM enzymes in excess. At faster growth rates, cells forsake these reserves in CCM in order to express more ribosomal proteins and enzymes in amino acid metabolism [25••], in line with the idea that protein synthesis regulates the cell growth rate. These results also highlight that the size of metabolic reserves can change depending on the growth rate, which means that proteome reserves must be quantified in the context of specific growth rates. Indeed, using an in silico approach to compare the amount of enzymes needed in computational simulations of cell growth and the experimentally measured

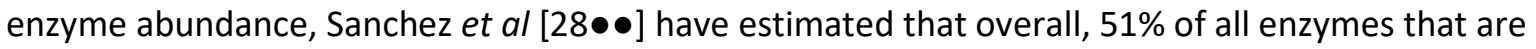
expressed in yeast are in active usage during typical laboratory growth (Fig 1C). Moreover, this approach allows enzyme usage to be estimated for each enzyme, which clearly shows that while most enzymes are synthesized in excess to maintain reserve capacities, a small amount of enzymes operate at or close to $100 \%$ capacity [ $28 \bullet \bullet$, indicating that these may be important rate-limiting control points that regulate the overall flux through the corresponding pathways. It can be anticipated that employing similar computational/experimental approaches in human cell models will allow the identification of similar points of control, which could represent potential drug targets in human diseases. Furthermore, this approach allows patient-specific enzyme abundance to be incorporated, which will enable the design and testing of personalized therapeutics against targeted enzymes.

\section{Metabolism}

The idea that replication of the proteome is a limiting factor in cell growth also manifests in the high energy cost of protein synthesis. In yeast, protein synthesis consumes $50-79 \%$ of energy generated in the cell [29-31], and the interaction between energy balance and protein synthesis gives rise to a trade-off between growth rate and biomass yield [31,32]. In human cells, dysregulation of metabolism and energy balance also underlies many diseases, including diabetes, mitochondrial diseases, and the Warburg effect in cancer. Since metabolism is a highly connected network of 
thousands of reactions, it is necessary not only to study the molecular mechanisms of individual enzymes and pathways, but also interactions between network components in the whole system. Genome-scale metabolic models (GEMs) represent one such framework of systems biology [33]. GEMs are complete metabolic maps representing the whole metabolic capacity of the cell or organism, where the stoichiometry and enzyme requirement of each metabolic reaction are recorded in silico. Today, comprehensive GEMs are available for a large number of organisms, including both yeast $[34,35 \bullet \bullet]$ and human cells $[36,37]$. Many human GEMs that are specific to certain tissue types or cancer cell lines are also available and have been used to study diseases and identify therapeutic targets [38,39].

With the rapid development of omics techniques, recently it has become of interest to integrate omics-level data into the GEM framework, which will allow points of metabolic flux control to be identified. Two general approaches have been used for this endeavour with yeast GEM as a scaffold of metabolic analysis. In the first approach, yeast GEM is used as-is to simulate metabolic flux at a given experimental condition, and how flux through individual enzymes or pathways is controlled can be inferred by comparing the simulated flux with the experimentally measured transcriptomic, proteomic, and/or phospho-proteomic levels (Fig 2A-B). Through this approach it was found that changes in the mRNA abundance of mitochondrial respiration proteins correlates well with changes in flux, while flux through the glycolytic pathway is not correlated with either the mRNA abundance or the protein abundance of glycolytic enzymes $[18 \bullet \bullet, 40]$. Instead, glycolysis appears to be engaged in complex regulation circuits involving a combination of substrate concentrations, enzyme allostery,

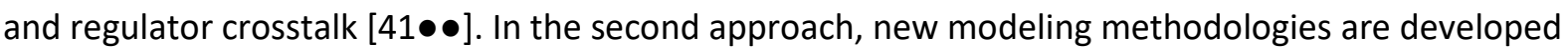
to incorporate omics data into GEM simulations themselves. For example, two studies $[28 \bullet \bullet, 42]$ have directly incorporated experimentally-measured enzyme abundance into GEM simulations, called enzyme-constrained GEM (ecGEM). In ecGEM, the simulated rate of each reaction cannot exceed an upper limit defined by the abundance and the turnover number $k_{c a t}$ of the catalyzing enzyme (Fig 2A-B). Through this modeling technique it was found that the Crabtree effect in yeast, analogous to the Warburg effect in human cancer cells [43], is determined by enzyme constraint $[28 \bullet \bullet, 42]$ : since enzymes in glycolysis and fermentation are smaller and more catalytically efficient compared to enzymes in the TCA cycle and ETC components, at high growth rates cells will trade off the bulky respiratory machinery for fermentation machinery, which has a smaller proteome cost and therefore enables cells to allocate more protein to translation, ie ribosomes, which is necessary for increasing its growth rate $[28 \bullet \bullet, 42]$. In a similar approach as direct incorporation of proteomics data in yeast GEM, a number of studies have directly incorporated metabolomics data into models of metabolic subsystems in yeast (Fig 2A-B) [44-46], although to date this approach has not been used 
genome-wide. This is in part due to the difficulty in obtaining metabolomics data at high quality and high coverage, but rapidly improving technologies will soon allow these limitations to be lifted [33]. In this modeling concept, the rate of a reaction is estimated based on the Gibbs free energy of the reaction, which is in turn regulated by metabolite concentrations and thermodynamic equilibrium

170 [44-46]. Indeed it has been shown that for most metabolic reactions, calculated flux is better correlated with metabolite concentrations than enzyme abundance $[47 \bullet \bullet]$. Thus, simulations with this modeling concept allows the identification of key flux-controlling metabolites as biomarkers, as exemplified in the identification of cytosolic-mitochondrial NAD $/ N A D H$ ratio as a key regulator of the ethanol-acetaldehyde redox shuttle [46]. In the near future it can be anticipated that multiple layers of omics data will be simultaneously incorporated into the GEM framework to allow for more sophisticated modeling of complex cellular behaviours. Since most yeast proteins and metabolic pathways have homologous counterparts in human cells, these insights obtained in yeast can be readily translated or verified in human cell models of pathophysiology in metabolic diseases.

\section{Gene expression}

180 Nearly all diseases are associated with changes in gene expression, either as a driver of disease onset and progression, or as a part of the cellular response to the diseased state. Gene expression changes in multifactorial diseases have received wide attention in clinical research to identify potential targets for clinical intervention. This was pioneered by transcriptomic profiling and is now pushing the bounds of personalized medicine through a rising capability to generate patient-specific datasets and lowering cost [48]. A major underlying assumption of these studies is that changes in transcriptlevel expression faithfully captures protein-level expression changes, which is the functional product of most genes. However, examinations of matching transcriptomic and proteomic datasets have indicated that this assumption is commonly violated, especially in complex and heterogeneous diseases such as cancer $[49,50]$. Recently several high-resolution datasets have been generated in yeast with matching transcriptomic and proteomic data across a large number of growth conditions or time series, which allowed the relationship between transcript and protein abundance to be closely examined, revealing several insights in the systems-level regulation of gene expression. In general, most transcripts are expressed at between 1-10 copies per cell, but protein abundance can range from 100 to $1 \mathrm{e} 6$ copies per cell $[18 \bullet \bullet, 51]$. This indicates that there is extensive control of protein expression at the translation step, and that large dynamic changes in transcript abundance will in most cases be buffered through this translation-level control to produce dampened effects at the protein level (Fig 3A). This appears to be also dependent on the metabolic context of the cell, as some environmental perturbations (eg salinity, temperature, EtOH stress) cause concerted changes in protein and transcript expression, indicative of near-constant and gene-specific translation 


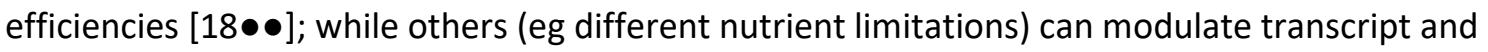

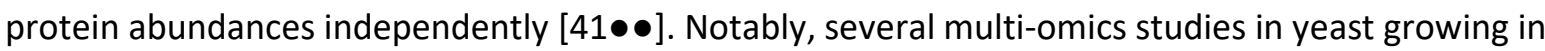
different contexts have identified two processes that are primarily regulated at the transcription

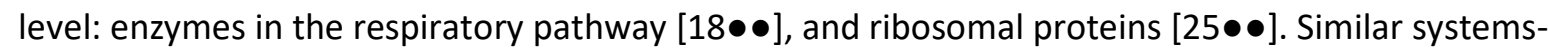
level identification of the control points of gene expression will have wide-spread implications in identification of actionable targets and development of therapeutics in human diseases and development of therapeutics.

Gene expression and metabolism also engage in complex reciprocal regulation, as exemplified in a number of subsystems such as the SNF1 (AMPK) and TOR (mTOR) regulation circuits $[1,6,52]$. Recently it has also become apparent that the metabolic context of the cell has global influences on gene expression beyond the classic feedback control systems. For example, up to $85 \%$ of the coding genome in yeast was found to be under epistatic regulation of leucine, histidine, uracil, and/or

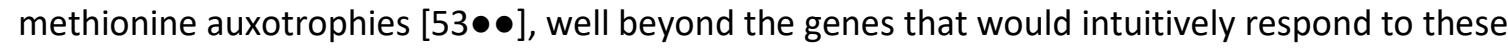
metabolic conditions such as enzymes in amino acid synthesis pathways. This represents a major caveat to the use of human cell models in the generation of multi-omics data for systems-level studies, since most cell lines are maintained in conditions that are not representative of the disease microenvironment, and the growth media is often supplemented with components (eg fetal bovine serum) whose exact chemical composition is unknown. Using yeast, it is possible to exert very fine control over both nutrient influx and cell growth, which facilitates modeling and hypothesis generation using high-resolution multi-omic datasets. One such example of this endeavour is the construction of a TRN network in order to delineate the relationship between gene expression and transcription factor (TF) binding in yeast. Preliminary studies mapping the genome-wide binding of

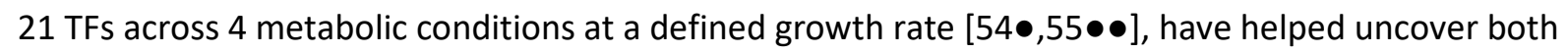
a threshold effect and a saturation effect of TF binding and transcript expression. The threshold effect indicates that a certain minimal level of TF binding must be met before transcription will respond, and the saturation effect indicates that there is a maximum level of transcriptional output

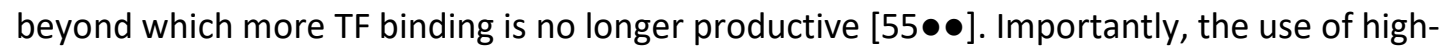
resolution regulomics data in yeast systems biology has allowed these biological phenomena to be quantified in a systematic way, which allows the effect of different TFs on the transcriptional output of a gene or gene set to be directly compared (Fig 3B). These insights can now be validated in human models with very simplistic experimental designs, which is of particular interest since transcription factors are common as both drivers of complex human diseases (eg c-Myc in various cancers) and as drug targets (eg steroid hormone receptors targeted by anti-inflammatory drugs), and to date a full functional accounting of the activities of these TFs remain incomplete. Validation of these insights in 
human models will further allow for precise therapeutic interventions to be designed, for example by CRISPR technology, to develop personalized medicine for disease treatment.

Yeast has been widely used as a model eukaryal organism, historically in molecular biology and now also in the rapidly developing field of systems biology. A number of yeast characteristics make it highly suitable for systems biology studies, notably the ability to exert fine control over the nutrient composition, metabolic flux, and growth rate of the cells. This has become especially important in the analysis of multi-omics datasets, not only because it removes these confounding variables thereby improving data quality, but also because orthogonal control of these variables allows new biological insights to be made. An elegant example of this is a recent study of cell cycle progression through the G1 checkpoint Start [56•], using an experimental system which allowed orthogonal control of glycolytic flux in yeast cells in an otherwise unaltered nutrient environment. In this study, Litsios et al [56•] revealed that passage through Start is controlled by metabolic oscillations in G1, notably an increase in glycolytic flux, which is coupled to a transient pulse in protein production rate necessary for Start. These insights clearly highlight that while the three key aspects of cell biology cell growth, metabolism, and gene expression - are highly connected and act in a concerted manner to give rise to biological phenomena, using yeast systems biology it is possible to dissect their dynamics and interactions through careful manipulation of experimental conditions and data analysis (graphical abstract, left). With the high degree of conservation of key cellular processes between yeast and human cells, this further allows human cell models (including primary patient samples) to be used in a more effective manner in simple, well-designed validation experiments. For example, currently cancer cell metabolism is thought to promote oncogenesis via three main axes: bioenergetics, biosynthesis of macromolecules, and redox balance [57]. The results of Litsios et al [56•] suggest the possibility of a fourth route of impact of high glycolytic flux on cancer cell growth, by directly driving cell cycle progression through the restriction point (the mammalian equivalent of Start in yeast). This now becomes a relatively simple hypothesis that can be tested in human cancer cell models, and if validated could significantly improve our understanding of cancer as a complex disease (graphical abstract, right). Thus, we anticipate that generation and analysis of highresolution multi-omics big data in yeast will continue to pioneer systems biology, generating new tools and concepts to accelerate our understanding of the mechanisms of complex human diseases. 

None.

267

\section{References and recommended reading}

Papers of particular interest, published within the period of review, have been highlighted as:

- of special interest

$\bullet \bullet$ of outstanding interest

\section{Figure captions}

Fig 1. Principles of cell growth. (A) Replication of a cell system is limited by the component that is replicated the slowest, which is typically proteins. P, protein. R, RNA. Asterisk indicates the ratelimiting process. (B) Under conditions where phosphate (a critical component of nucleotides) is limiting, RNA becomes the component that is replicated the slowest and the cell growth rate becomes dependent on the RNA transcription rate. $P_{i}$, phosphate. (C) The proteome reserve of a typical yeast cell is approximately $50 \%$ but varies depending on the growth condition. Arrowheads represent changes in growth condition.

Fig 2. Use of GEM scaffold for integrative omics data analysis. (A) An example of a reaction in a GEM catalyzed by an enzyme. (B) Mathematical models and omics data analysis approaches for the represented reaction. $r$, rate of reaction. Asterisk indicates that various measurements of the enzyme can be used: eg mRNA abundance, protein abundance, PTMs, or experimentally measured flux through the enzyme. $k_{c a t}$, enzyme turnover number. $\sigma$, enzyme usage. $\Delta r G^{0}$, standard Gibbs free energy of compound formation. $\mathrm{R}$, universal gas constant. $\mathrm{T}$, temperature.

Fig 3. Gene expression dynamics delineated by yeast systems biology. (A) There is extensive control of gene expression at the translation step which dampens the effect of transcript variability to the protein level. (B) The effect of TF binding on transcriptional output can be directly compared with the construction of TRN using high-resolution omics datasets. 


\section{References}

1. Nielsen J: Yeast systems biology: Model organism and cell factory. Biotechnol J (2019) e1800421.

2•. Barberis M, Todd RG, van der Zee L: Advances and challenges in logical modeling of cell cycle regulation: Perspective for multi-scale, integrative yeast cell models. FEMS Yeast Res (2017) 17(1).

A thorough review of multi-scale modeling of cell division and metabolism.

3. Falcone C, Mazzoni C: External and internal triggers of cell death in yeast. Cell Mol Life Sci (2016) 73(11-12):2237-2250.

4. Putnam CD, Kolodner RD: Pathways and mechanisms that prevent genome instability in Saccharomyces cerevisiae. Genetics (2017) 206(3):1187-1225.

5. Korber M, Klein I, Daum G: Steryl ester synthesis, storage and hydrolysis: A contribution to sterol homeostasis. Biochim Biophys Acta Mol Cell Biol Lipids (2017) 1862(12):1534-1545.

6. Gonzalez A, Hall MN: Nutrient sensing and tor signaling in yeast and mammals. EMBO J (2017) 36(4):397-408.

7. Malina C, Larsson C, Nielsen J: Yeast mitochondria: An overview of mitochondrial biology and the potential of mitochondrial systems biology. FEMS Yeast Res (2018) 18(5).

8. Kapahi $\mathrm{P}$, Kaeberlein M, Hansen M: Dietary restriction and lifespan: Lessons from invertebrate models. Ageing Res Rev (2017) 39(3-14).

9. Seynnaeve D, Vecchio MD, Fruhmann G, Verelst J, Cools M, Beckers J, Mulvihill DP, Winderickx J, Franssens V: Recent insights on alzheimer's disease originating from yeast models. Int J Mol Sci (2018) 19(7).

10. Rajakumar T, Munkacsi AB, Sturley SL: Exacerbating and reversing lysosomal storage diseases: From yeast to humans. Microb Cell (2017) 4(9):278-293.

11. Petranovic $D$, Nielsen J: Can yeast systems biology contribute to the understanding of human disease? Trends Biotechnol (2008) 26(11):584-590.

12. Kafri M, Metzl-Raz E, Jonas F, Barkai N: Rethinking cell growth models. FEMS Yeast Res (2016) 16(7).

13. Sherman F: Getting started with yeast. Methods Enzymol (2002) 350(3-41). 
14. Scott M, Klumpp S, Mateescu EM, Hwa T: Emergence of robust growth laws from optimal regulation of ribosome synthesis. Mol Syst Biol (2014) 10(747).

15. Koch AL: Why can't a cell grow infinitely fast? Can J Microbiol (1988) 34(4):421-426.

16. Shachrai I, Zaslaver A, Alon U, Dekel E: Cost of unneeded proteins in E. coli is reduced after several generations in exponential growth. Mol Cell (2010) 38(5):758-767.

17. Bosdriesz E, Molenaar D, Teusink B, Bruggeman FJ: How fast-growing bacteria robustly tune their ribosome concentration to approximate growth-rate maximization. FEBS J (2015) 282(10):2029-2044.

18••. Lahtvee PJ, Sanchez BJ, Smialowska A, Kasvandik S, Elsemman IE, Gatto F, Nielsen J: Absolute quantification of protein and mrna abundances demonstrate variability in genespecific translation efficiency in yeast. Cell Syst (2017) 4(5):495-504 e495.

A high-resolution multi-omics dataset of matching yeast transcriptomics, proteomics, protein degradation rate, and protein synthesis rate, at a defined media composition and constant growth rate.

19. Geisberg JV, Moqtaderi Z, Fan X, Ozsolak F, Struhl K: Global analysis of mrna isoform halflives reveals stabilizing and destabilizing elements in yeast. Cell (2014) 156(4):812-824.

20. Pelechano V, Chavez S, Perez-Ortin JE: A complete set of nascent transcription rates for yeast genes. PLoS One (2010) 5(11):e15442.

21••. Kafri M, Metzl-Raz E, Jona G, Barkai N: The cost of protein production. Cell Rep (2016) 14(1):22-31.

A careful analysis of the role of mRNA and protein synthesis in controlling the cell growth rate, indicating that either mRNA synthesis or protein synthesis can be the limiting factor of cell growth rate, depending on the growth condition.

22. Christiano R, Nagaraj N, Frohlich F, Walther TC: Global proteome turnover analyses of the yeasts S. cerevisiae and S. pombe. Cell Rep (2014) 9(5):1959-1965.

23. Tirosh I, Reikhav S, Levy AA, Barkai N: A yeast hybrid provides insight into the evolution of gene expression regulation. Science (2009) 324(5927):659-662.

24. Dori-Bachash $\mathrm{M}$, Shema $\mathrm{E}$, Tirosh I: Coupled evolution of transcription and mRNA degradation. PLOS Biol (2011) 9(7):e1001106.

25••. Metzl-Raz E, Kafri M, Yaakov G, Soifer I, Gurvich Y, Barkai N: Principles of cellular resource allocation revealed by condition-dependent proteome profiling. Elife (2017) 6.

Showing that cells maintain a certain amount of excess ribosomes $-8 \%$ in yeast. 
26•. Mori M, Schink S, Erickson DW, Gerland U, Hwa T: Quantifying the benefit of a proteome reserve in fluctuating environments. Nat Commun (2017) 8(1):1225.

Showing that the amount of excess ribosomes expressed is evolutionarily selected for.

27. Muller PY, Milton MN: The determination and interpretation of the therapeutic index in drug development. Nat Rev Drug Discov (2012) 11(10):751-761.

28••. Sanchez BJ, Zhang C, Nilsson A, Lahtvee PJ, Kerkhoven EJ, Nielsen J: Improving the phenotype predictions of a yeast genome-scale metabolic model by incorporating enzymatic constraints. Mol Syst Biol (2017) 13(8):935.

Development of ecYeast GEM where flux is constrained by enzyme abundance.

29. Dacheux E, Firczuk H, McCarthy JE: Rate control in yeast protein synthesis at the population and single-cell levels. Biochem Soc Trans (2015) 43(6):1266-1270.

30. Liu Y, Beyer A, Aebersold R: On the dependency of cellular protein levels on mRNA abundance. Cell (2016) 165(3):535-550.

31. Hong KK, Hou J, Shoaie S, Nielsen J, Bordel S: Dynamic 13C-labeling experiments prove important differences in protein turnover rate between two Saccharomyces cerevisiae strains. FEMS Yeast Res (2012) 12(7):741-747.

32. Canelas AB, Harrison N, Fazio A, Zhang J, Pitkanen JP, van den Brink J, Bakker BM, Bogner L, Bouwman J, Castrillo JI, Cankorur A, Chumnanpuen P, Daran-Lapujade P, Dikicioglu D, van Eunen K, Ewald JC, Heijnen JJ, Kirdar B, Mattila I, Mensonides FI, Niebel A, Penttila M, Pronk JT, Reuss M, Salusjarvi L, Sauer U, Sherman D, Siemann-Herzberg M, Westerhoff H, de Winde J, Petranovic D, Oliver SG, Workman CT, Zamboni N, Nielsen J: Integrated multilaboratory systems biology reveals differences in protein metabolism between two reference yeast strains. Nat Commun (2010) 1(145).

33. Nielsen J: Systems biology of metabolism. Annu Rev Biochem (2017) 86(245-275).

34. Forster J, Famili I, Fu P, Palsson BO, Nielsen J: Genome-scale reconstruction of the Saccharomyces cerevisiae metabolic network. Genome Res (2003) 13(2):244-253.

35••. Lu H, Li F, Sanchez BJ, Zhu Z, Li G, Domenzain I, Marcisauskas S, Anton PM, Lappa D, Lieven C, Beber ME, Sonnenschein N, Kerkhoven EJ, Nielsen J: A consensus S. cerevisiae metabolic model Yeast8 and its ecosystem for comprehensively probing cellular metabolism. Nat Commun (2019) 10(1):3586.

Latest release of Yeast GEM including the reconstructed metabolic network of 1,011 S. cerevisiae strains and a database containing 3D structures of metabolic proteins. 
36. Duarte NC, Becker SA, Jamshidi N, Thiele I, Mo ML, Vo TD, Srivas R, Palsson BO: Global reconstruction of the human metabolic network based on genomic and bibliomic data. Proc Natl Acad Sci U S A (2007) 104(6):1777-1782.

37. Swainston N, Smallbone K, Hefzi H, Dobson PD, Brewer J, Hanscho M, Zielinski DC, Ang KS, Gardiner NJ, Gutierrez JM, Kyriakopoulos S, Lakshmanan M, Li S, Liu JK, Martinez VS, Orellana CA, Quek LE, Thomas A, Zanghellini J, Borth N, Lee DY, Nielsen LK, Kell DB, Lewis NE, Mendes P: Recon 2.2: From reconstruction to model of human metabolism. Metabolomics (2016) 12(109).

38. Shlomi T, Cabili MN, Herrgard MJ, Palsson BO, Ruppin E: Network-based prediction of human tissue-specific metabolism. Nat Biotechnol (2008) 26(9):1003-1010.

39. Agren R, Bordel S, Mardinoglu A, Pornputtapong N, Nookaew I, Nielsen J: Reconstruction of genome-scale active metabolic networks for 69 human cell types and 16 cancer types using INIT. PLoS Comput Biol (2012) 8(5):e1002518.

40. Bordel S, Agren R, Nielsen J: Sampling the solution space in genome-scale metabolic networks reveals transcriptional regulation in key enzymes. PLoS Comput Biol (2010) 6(7):e1000859.

41••. Hackett SR, Zanotelli VR, Xu W, Goya J, Park JO, Perlman DH, Gibney PA, Botstein D, Storey JD, Rabinowitz JD: Systems-level analysis of mechanisms regulating yeast metabolic flux. Science (2016) 354(6311).

Another high-resolution multi-omics dataset of matching yeast enzyme concentrations, metabolite concentrations, and calculated metabolic fluxes, at several defined growth media compositions and controlled growth rate.

42. Nilsson A, Nielsen J: Metabolic trade-offs in yeast are caused by F1F0-ATP synthase. Sci Rep (2016) 6(22264).

43. Shlomi T, Benyamini T, Gottlieb E, Sharan R, Ruppin E: Genome-scale metabolic modeling elucidates the role of proliferative adaptation in causing the warburg effect. PLoS Comput Biol (2011) 7(3):e1002018.

44. Soh KC, Miskovic L, Hatzimanikatis V: From network models to network responses: Integration of thermodynamic and kinetic properties of yeast genome-scale metabolic networks. FEMS Yeast Res (2012) 12(2):129-143.

45. Birkenmeier M, Mack M, Roder T: A coupled thermodynamic and metabolic control analysis methodology and its evaluation on glycerol biosynthesis in Saccharomyces cerevisiae. Biotechnol Lett (2015) 37(2):307-316. 
46. Jol SJ, Kummel A, Terzer M, Stelling J, Heinemann M: System-level insights into yeast metabolism by thermodynamic analysis of elementary flux modes. PLoS Comput Biol (2012) 8(3):e1002415.

47••. Zelezniak A, Vowinckel J, Capuano F, Messner CB, Demichev V, Polowsky N, Mulleder M, Kamrad S, Klaus B, Keller MA, Ralser M: Machine learning predicts the yeast metabolome from the quantitative proteome of kinase knockouts. Cell Syst (2018) 7(3):269-283 e266.

A machine learning framework to understand reciprocal regulation of gene expression and metabolism.

48. Rabbani B, Nakaoka H, Akhondzadeh S, Tekin M, Mahdieh N: Next generation sequencing: Implications in personalized medicine and pharmacogenomics. Mol Biosyst (2016) 12(6):1818-1830.

49. Zhang B, Wang J, Wang X, Zhu J, Liu Q, Shi Z, Chambers MC, Zimmerman LJ, Shaddox KF, Kim S, Davies SR, Wang S, Wang P, Kinsinger CR, Rivers RC, Rodriguez H, Townsend RR, Ellis MJ, Carr SA, Tabb DL, Coffey RJ, Slebos RJ, Liebler DC, NCI CPTAC Investigators: Proteogenomic characterization of human colon and rectal cancer. Nature (2014) 513(7518):382-387.

50. Tyanova S, Albrechtsen R, Kronqvist P, Cox J, Mann M, Geiger T: Proteomic maps of breast cancer subtypes. Nat Commun (2016) 7(10259).

51. Marguerat S, Schmidt A, Codlin S, Chen W, Aebersold R, Bahler J: Quantitative analysis of fission yeast transcriptomes and proteomes in proliferating and quiescent cells. Cell (2012) 151(3):671-683.

52. Mihaylova MM, Shaw RJ: The ampk signalling pathway coordinates cell growth, autophagy and metabolism. Nat Cell Biol (2011) 13(9):1016-1023.

53••. Alam MT, Zelezniak A, Mulleder M, Shliaha P, Schwarz R, Capuano F, Vowinckel J, Radmanesfahar E, Kruger A, Calvani E, Michel S, Borno S, Christen S, Patil KR, Timmermann $B$, Lilley KS, Ralser M: The metabolic background is a global player in saccharomyces gene expression epistasis. Nat Microbiol (2016) 1(15030).

A systematic examination of the interaction between gene expression and metabolism showing that a precise perturbation in metabolism can lead to system-wide consequences in gene expression responses.

54•. Bergenholm D, Liu G, Holland P, Nielsen J: Reconstruction of a global transcriptional regulatory network for control of lipid metabolism in yeast by using chromatin immunoprecipitation with lambda exonuclease digestion. mSystems (2018) 3(4). Showing that transcription factors in yeast engage in combinatorial and complementary regulation of gene expression which is dependent on the cell metabolic state. 
55••. Holland P, Bergenholm D, Borlin CS, Liu G, Nielsen J: Predictive models of eukaryotic transcriptional regulation reveals changes in transcription factor roles and promoter usage between metabolic conditions. Nucleic Acids Res (2019) 47(10):4986-5000.

A high-resolution dataset of transcription factor binding and gene expression of yeast growing in 4 different media compositions at a constant growth rate; a transparent modeling framework to describe and predict the contribution of each TF to gene expression.

56•. Litsios A, Huberts Dhew, Terpstra HM, Guerra P, Schmidt A, Buczak K, Papagiannakis A, Rovetta M, Hekelaar J, Hubmann G, Exterkate M, Milias-Argeitis A, Heinemann M: Differential scaling between $\mathrm{G} 1$ protein production and cell size dynamics promotes commitment to the cell division cycle in budding yeast. Nat Cell Biol (2019) 21(11):13821392.

Showing that sufficient flux through the central carbon metabolism drives a transient pulse in protein production in $\mathrm{G} 1$ which is essential for passage through Start, using an elegant experimental system that allows glycolytic flux in yeast cells to be orthogonally controlled.

57. DeBerardinis RJ, Chandel NS: Fundamentals of cancer metabolism. Sci Adv (2016) 2(5):e1600200. 
524 Figure 1
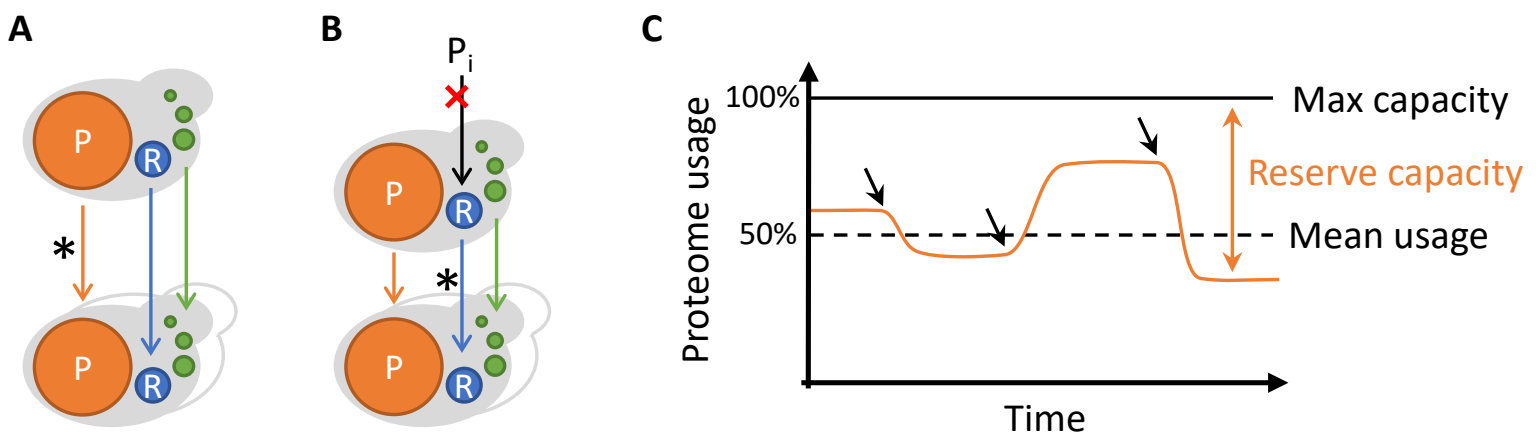

526

527 
528 Figure 2

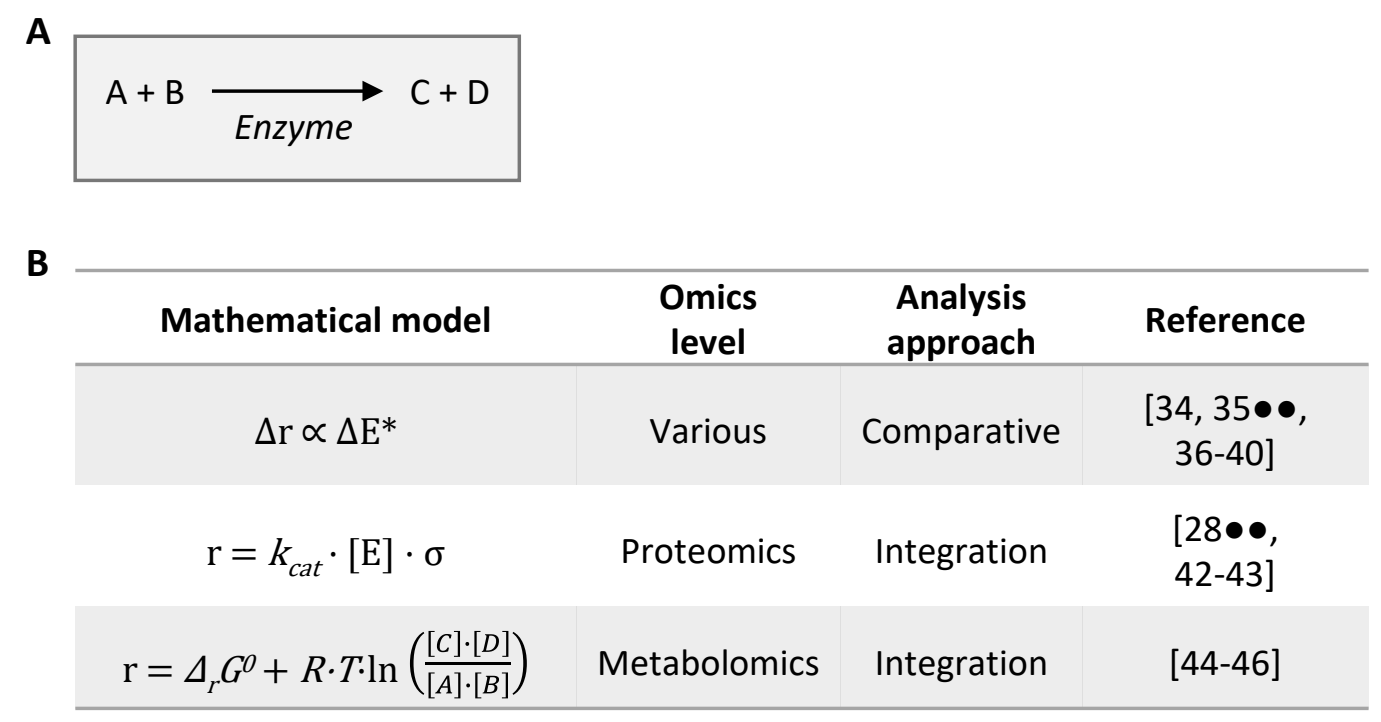


531 Figure 3

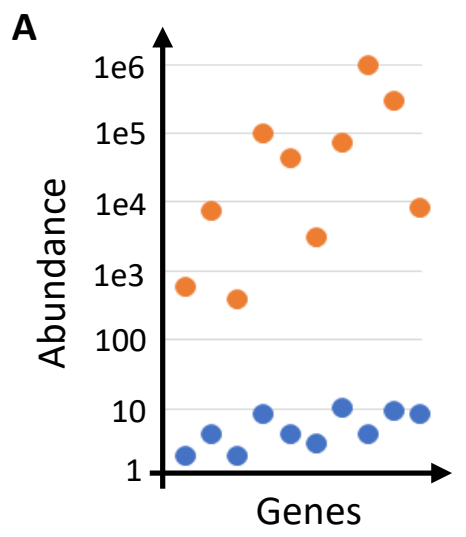

$$
\begin{aligned}
& \text { protein expression unlikely } \\
& \text { to change by }>2 \text {-fold }
\end{aligned}
$$

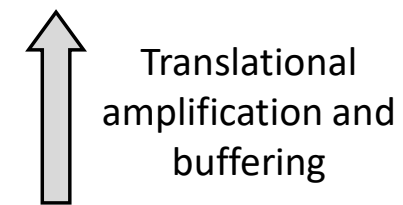

mRNA expression can

change by several fold

B

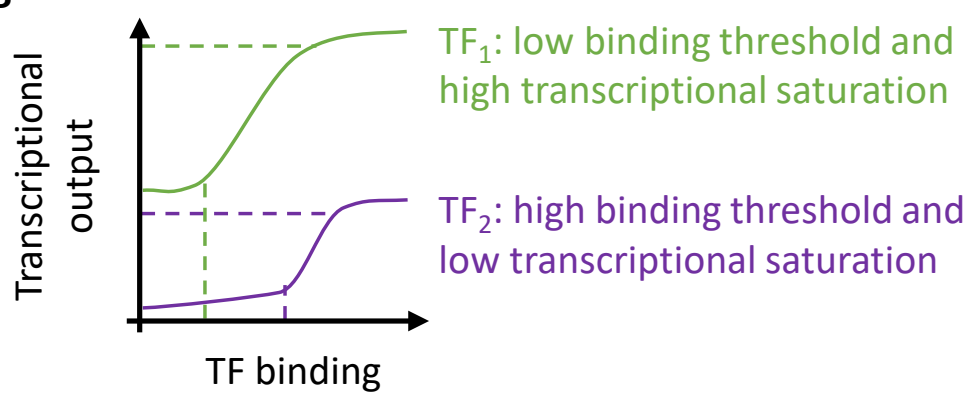

